chain. However, typical amide-I absorption peaks imply a lifetime of about $1 \mathrm{ps, \text {which }}$ is too short for normal biological mechanisms. Davydov suggested that this lifetime could be markedly increased if the excitation interacted nonlinearly with other excitations on the polypeptide chain. In particular, the localized amide-I bond energy could act as a source for phonon (sound) waves excited along the chain, which in turn could act as a potential well to trap the bond energy and prevent its dispersion. The complete excitation would therefore be localized and inherently nonlinear in nature, that is it would be a soliton. Scott and his colleagues have spent several years studying and extending Davydov's model. Recently, they have used it to interpret spectral lines measured in Laser-Raman studies of metabolically active Escherichia coli (Scott, A.C. Phys. Rev. $A$ 26, 578; 1982), arguing that such spectra are consistent with the notion of soliton excitation along the chain. Scott also presented evidence of self-trapping of amide-I vibrational energy in crystalline acetanilide, which can be similarly explained in terms of the Davydov soliton excitation hypothesis.
Returning us to the original hunting ground for solitons-fluid mechanicsJ.B. Keller (Stanford University) described the way in which solitons are generated and propagate in terms of initial value and initial boundary-value problems. Experiments in which a ship is pulled along a narrow test tank demonstrate soliton excitation. A critical parameter $F_{R}$ (ship speed/speed of long-wavelength, smallamplitude waves) exists, such that solitons are excited only when $F_{R}>1$. Because of the walls of the test tank, the solitions propagate ahead of the ship-hence the need to model the system as an initial boundaryvalue problem rather than simply as an initial value problem in studies on the soliton properties of the Korteweg deVries equation, for example.

Seven other papers reflected the rich variety of topics to which solitons have relevance and also dealt with the more abstract and mathematical topics of algebraic and topological structure of the soliton equations themselves.

John Elgin is in the Department of Mathematics, Imperial College of Science and Technology, London SW7 2BZ, UK.

\title{
High-pressure physics
}

\section{Doubly-shocked helium}

\section{from P.V.E. McClintock}

IN EXPERIMENTS carried out by W.J. Nellis and colleagues at Lawrence Livermore National Laboratory in California, and reported in Physical Review Letters 53, $1248 ; 1984$, liquid helium has been shockcompressed to densities of up to five times that of the normal liquid. The pressure transiently achieved in the experiments approaches that to which helium is subjected on some planets.

The behaviour of matter at extreme densities is of particular interest not only for its own sake but also because of its relevance to an understanding of the giant planets. Saturn, for example, appears to have an internal energy source of some kind and it is possible that the unmixing and gravitational separation of the hydrogenhelium fluid is responsible for the evolution of heat. Whether such phase separation occurs in reality is not at all easy to resolve by laboratory experiments because of the extreme pressures and temperatures on Saturn, which are believed to range up to about 1 terrapascal $\left(10^{7}\right.$ atmospheres) and $14,000 \mathrm{~K}$ respectively. To approach the requisite pressure a number of powerful compression methods are being developed. These include static compression techniques, the controlled use of explosives and the operation of a two-stage light-gas gun, as used by Nellis et al.

The light-gas gun, which can be considered as a quite monstrous analogue of an airgun, is used to fire a flat-ended projectile at very high velocity on to the sample under study, which is thereby transiently compressed. To maximize the initial, and thus also the final, densities the sample is usually chosen to be in its solid or-as in the present case-liquid phase. The process is initiated by the firing of a charge of gunpowder in a combustion chamber. That drives a piston up a gun barrel of relatively large diameter, and the piston very rapidly compresses hydrogen gas into a second and much narrower barrel, along which the projectile is propelled at a large and approximately constant acceleration, finally emerging from the muzzle at a velocity of between 3 and $7 \mathrm{~km}^{-1}$. When the projectile strikes the outside of the sample holder, a shock wave is generated and propagates through into the liquid helium, carrying with it a region of extreme pressure. As a result, there are almost instantaneous and dramatic increases in the temperature and pressure of the helium, which are further increased when the shock wave reflects from the rear surface of the sample holder.

Notwithstanding the extreme (and destructive) speed with which these events occur, it is still possible to acquire detailed information about the state of the sample during the shock. The velocity of the projectile before impact is determined precisely by means of a flash X-ray technique, and the velocity of the shock waves is measured from their transit times between self- shorting coaxial metal pins (relying on the fact that many materials that are normally insulators become conductors under conditions of extreme pressure). From a knowledge of the initial density of the liquid helium and the characteristics of the projectile and sample chamber it is possible to calculate the shock pressure, density and temperature by use of the so-called Rankine-Hugoniot equations. By such means, Nellis et al. calculate that the first shock transiently increases the temperature of the helium from $4.3 \mathrm{~K}$ to $12,000 \mathrm{~K}$ and its pressure from just above atmospheric to 16 gigapascals. After reflection of the shock wave, the 'double shock' takes the temperature to $21,000 \mathrm{~K}$ and the pressure to 56 gigapascals.

The results are compared with some theoretical predictions in the figure. Each of the broken curves is derived from a different helium interatomic potential based on past work. Only the best of these (dashed curve) has been extended to pressures above that of the first shock, since it is immediately evident that the others are in serious disagreement with experiment. The full curve represents an improved version of the theory shown by the dashed curve, adjusted in the light of the singleshock results; and the open circle represents the resultant prediction of where the double-shock data point ought to fall. It is, in fact, in excellent agreement with prediction, which can be regarded as reassuring evidence in support of the self-consistency of the data analysis and the accuracy of the adjusted interatomic potential.

Although the pressures achieved are still not up to the highest pressures found in the

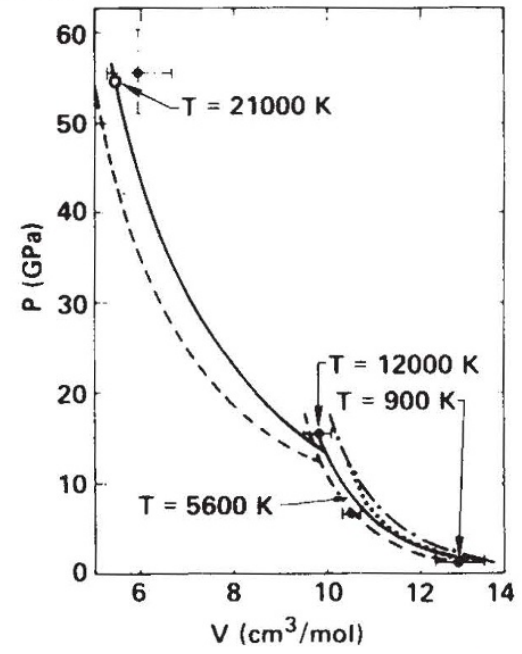

Comparison of shock-compression results for helium (points with error bars) with theoretical predictions (curves), the pressure $P$ being plotted as a function of molar volume V. From Nellis, W.J. et al. Phys. Rev. Letters. 53, 1248; 1984. giant planets, they are getting remarkably close, so laying a firm foundation of experimental data for the building of theories to describe those distant worlds.

P.V.E. McClintock is in the Department of Physics, University of Lancaster, Lancaster LAI $4 Y B, U K$. 